\title{
Intrahepatic Cholangiocarcinoma Involving the Hepatocaval Confluence with Bile Duct Tumor Thrombus Mimicking a Klatskin Tumor: A Case Report
}

\author{
Florin Botea ${ }^{1}$, Alexandru Barcu ${ }^{1}$, Nausica Picu ${ }^{1}$, Ruxandra Fota $^{2}$, Irinel Popescu ${ }^{1}$
}

1"Dan Setlacec" Center of General Surgery and Liver Transplantation, Fundeni Clinical Institute, Bucharest, Romania

${ }^{2}$ Intensive Care Unit, Fundeni Clinical Institute, Bucharest, Romania

\author{
Corresponding author: \\ Irinel Popescu, MD, PhD, FACS, FEBS \\ Center of General Surgery and Liver \\ Transplantation, Fundeni Clinical Institute \\ Fundeni Street no. 258, 022328, \\ Bucharest, Romania \\ E-mail: irinel.popescu220@gmail.com
}

\section{ABSTRACT}

Background: Intrahepatic cholangiocarcinoma (ICC) is a rare malignant tumor arising from the epithelial cells of the intrahepatic bile ducts. The aim of the present paper is to report a rare case of centrally located ICC involving the hepatocaval confluence and generating a tumor thrombus in the biliary duct of segment 4 expanding into the left hepatic duct and main common duct, mimicking a type IIIB Klatskin tumor.

Case presentation: A 66-year old female presented for epigastric abdominal pain and weight loss, with cytolysis (ALAT= $323 \mathrm{U} /$; normal ASAT), high GGT (618 U/L), but normal phosphatase alkaline and bilirubin levels; CA 19-9 was elevated (257 U/mL), while CEA was normal. At dual-phase multi-detector CT and magnetic resonance cholangiopancreatography, a simultaneous ICC and type IIIB perihilar cholangiocarcinoma (Klatskin tumor) were diagnosed. Intraoperatively, the ICC located in segments 1, 4 and 8, infiltrating the left and middle hepatic veins and in contact with the right hepatic vein was confirmed, while the hilar lesion proved to be a bile duct tumor thrombus originating from the ICC. Consequently, a left hepatectomy extended to segments 1 and 8 with hilar approach, with en-bloc resection of main biliary duct, and hilar lymph node dissection was performed. The right hepatic vein and an accessory middle hepatic vein were preserved, accepting 0 -mm resection margin at this level. The postoperative outcome was remarkably uneventful.

Conclusion: Locally advanced ICC is a challenging presentation for both diagnosis and treatment, for which complex major liver resection is effective when performed in a high volume HPB center.

Key words: intrahepatic cholangiocarcinoma, bile duct tumor thrombus, major liver resection

\section{BACKGROUND}

Intrahepatic cholangiocarcinoma (ICC) is a rare malignant tumor arising from the epithelial cells of the intrahepatic bile ducts. The incidence of ICC has been continuously rising over the past decades worldwide, which may be related to the increase of certain risk factors such as cirrhosis, alcoholic liver disease, and hepatitis $C$ virus infection (1). Contrarily, the incidence of the extrahepatic type 
is declining (2). Liver resection is the main option for treatment in patients with disease limited to the liver provided that future liver remnant liver is insured. However, true resectability can often only be assessed during surgery.

The aim of the present paper is to report a rare case of centrally located ICC involving the hepatocaval confluence and generating a tumor thrombus in the biliary duct of segment 4 expanding into the left hepatic duct and main common duct, mimicking a type IIIB Klatskin tumor.

\section{CASE PRESENTATION}

A 66-year old female, with no significant history, presented for epigastric abdominal pain and weight loss. A 66-year old female presented for epigastric abdominal pain and weight loss, with cytolysis (ALAT= $323 \mathrm{U} / \mathrm{l}$; normal ASAT), high GGT (618 U/L), but normal phosphatase alkaline and bilirubin levels; the serum carbohydrate antigen level (CA 19-9) was elevated (257 $\mathrm{U} / \mathrm{mL}$ ), and the carcinoembryonic antigen (CEA) was normal $(1.4 \mathrm{ng} / \mathrm{mL})$.

Transabdominal ultrasound detected an obstruction of the main biliary confluence with significant intrahepatic biliary tract dilatation. Dual-phase multidetector CT reported a hypodense mass with regular margins at the level of the left and main hepatic duct, with significant intrahepatic biliary tract dilatation, with consequent atrophy of the left hemiliver (fig. 1); no intrahepatic mass was reported. At magnetic resonance cholangiopancreatography (MRCP), an intrahepatic mass infiltrating the segments 1, 4 (quasi-completely) and 8 (partially), infiltrating the left intrahepatic biliary tract, middle hepatic veins and suspicion of infiltration of the right hepatic vein (RHV) was described (fig. 2); it also confirmed the presence of the nodular mass at the level of the main biliary confluence and left hepatic duct. Consequently, a simultaneous ICC and a type IIIB perihilar cholangiocarcinoma (Klatskin tumor) were
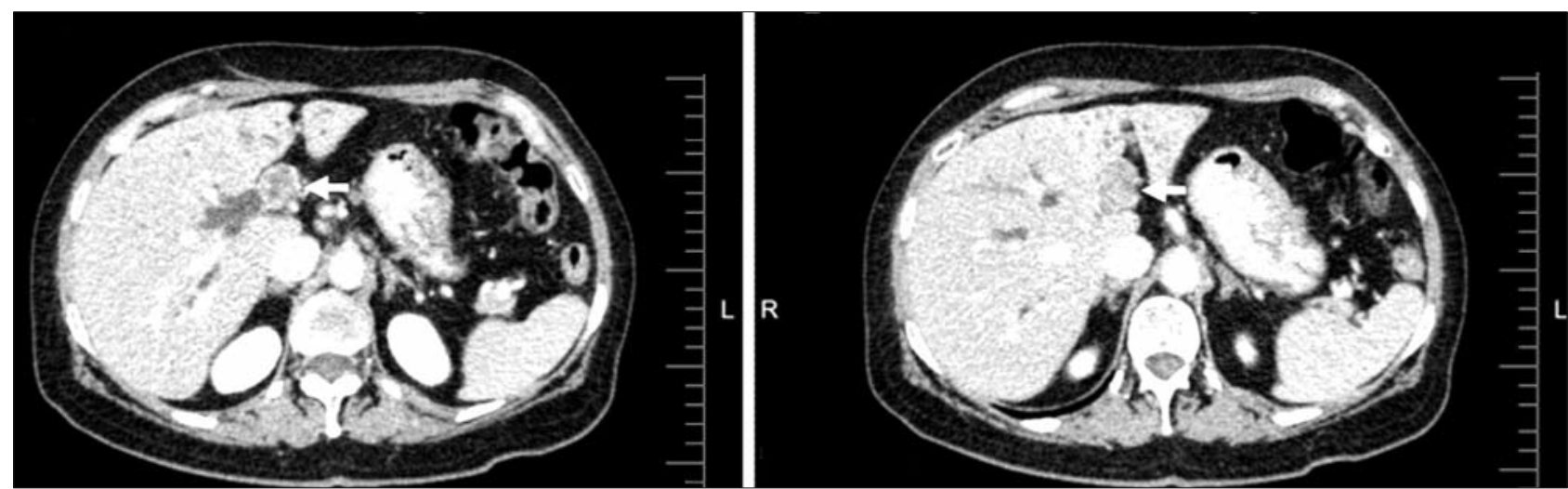

Figure 1 - Dual-phase multi-detector CT shows a hypodense mass with regular margins at the level of the left and main hepatic duct (arrow), with significant intrahepatic biliary tract dilatation with consequent atrophy of the left hemiliver (fig. 1); no intrahepatic mass was reported
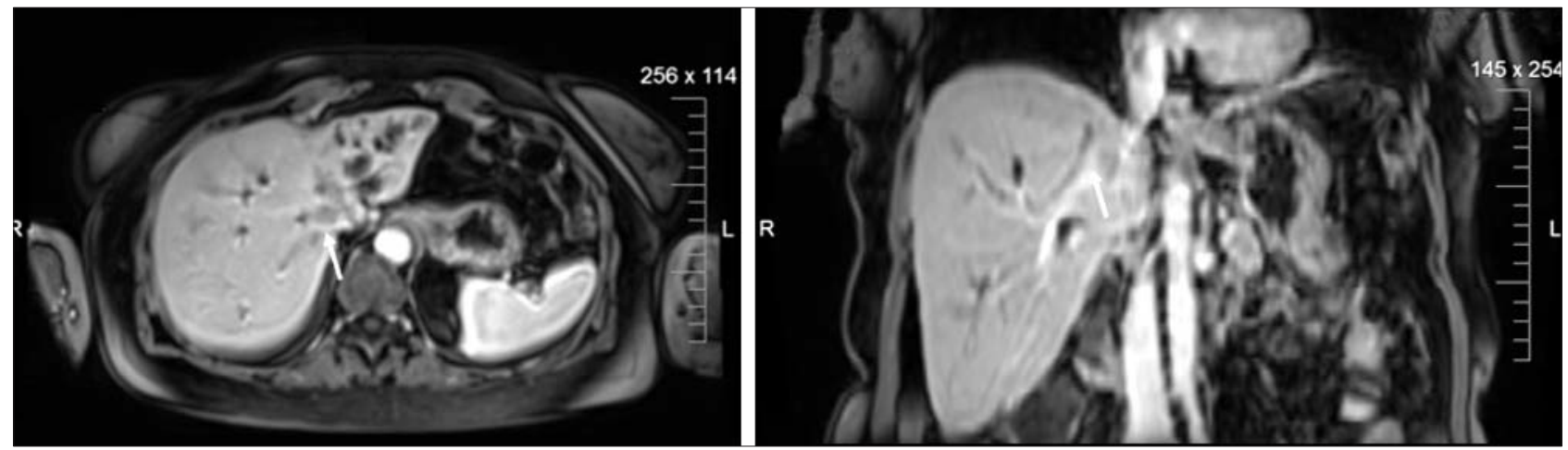

Figure 2 - Magnetic resonance cholangiopancreatography (MRCP) described an intrahepatic mass infiltrating the segments 1, 4 (quasi-completely) and 8 (partially), infiltrating the left intrahepatic biliary tract, middle hepatic veins and suspicion of infiltration of the right hepatic vein (RHV) (arrow). The mass was not detected at CT. 
diagnosed. No distant metastases were observed at the preoperative imaging examinations.

The patient was discussed at the multidisciplinary oncological board and referred for liver resection with potential reconstruction of the RHV, and was therefore scheduled for surgery.

At intraoperative ultrasound, the intrahepatic mass was proven to invade the common trunk of the left and middle hepatic veins and to be in contact with the RHV (without signs of tumor infiltration). Surprisingly, the perihilar lesion proved to be a bile duct tumor thrombus originating from the centrally located ICC. Consequently, a left hepatectomy extended to segments 1,5 and 8 using hilar approach, with en-bloc resection of main biliary duct, and hilar lymph node dissection was performed (figs. 3 and 4). Total vascular exclusion was deployed at the end of the hepatectomy for 10 minutes, during the tumor detachment from the RHV. The RHV along with a large accessory middle right hepatic vein were preserved by detaching them from the tumor, accepting 0-mm margins at this level. Limited resection of the anterior wall of the RHV at the level of the caval confluence was required; no patch was needed for its reconstruction (fig. 3).

The resection of the common bile duct and main biliary confluence was decided in order to excise en-bloc the biliary tumor thrombus, thus avoiding the risk of tumor cells spillage and consequent potential carcinomatosis after thrombectomy (the alternative to bile duct resection). The common bile duct was distally ligated prior to sectioning with the same intent to avoid tumor cells spillage. The decision to resect the main biliary confluence was also endorsed by intraoperative ultrasound, that depicted poor delineation between the tumor thrombus and the bile duct wall. This finding was proven on the resected specimen, in which the thrombus remained partially attached to the main biliary confluence (fig. 4). The biliary drainage was reconstructed using a Roux-en-Y cholangiojejunostomy.
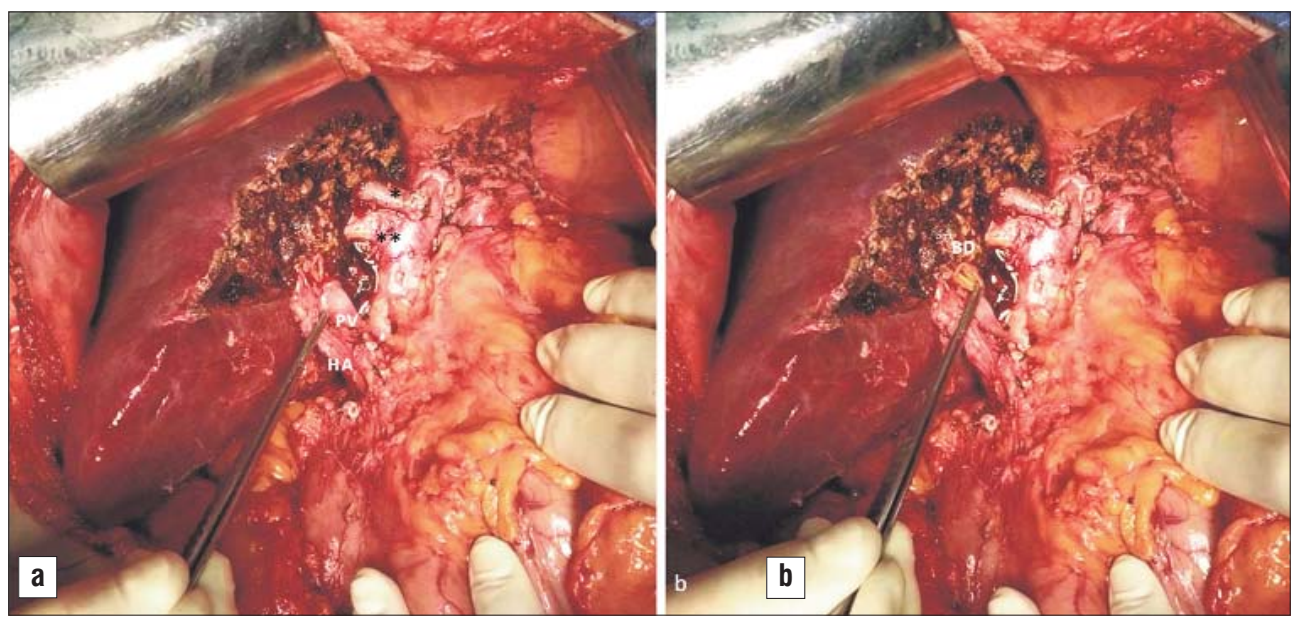

Figure 3 - Remnant liver (segments 6 and 7 and part of segment 5 and 8) with common and right hepatic vein $(\mathrm{HA})$, common and right portal vein (PV), stump of the right hepatic duct (BD), right hepatic vein $\left(^{*}\right)$, and accessory middle hepatic vein $\left({ }^{* *}\right)$.

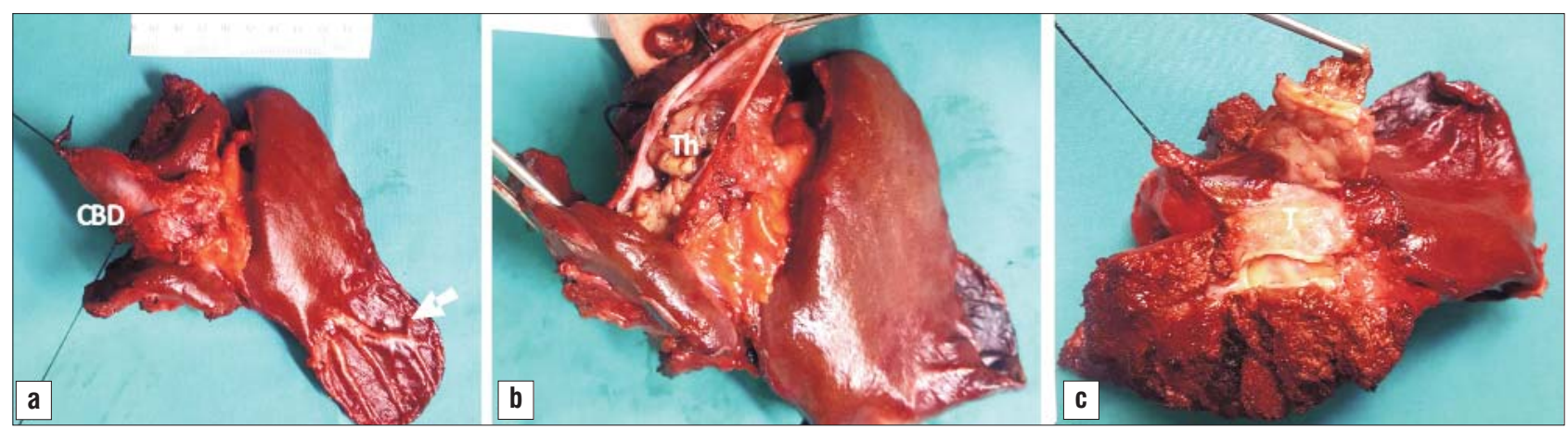

Figure 4 - Specimen showing the common bile duct (CBD) filled with the tumor thrombus, severe atrophy of the left lateral section (arrow), thrombus (Th) at the level of the main biliary confluence, and the intrahepatic tumor (T). 
The operative time was $360 \mathrm{~min}$. The intraoperative blood loss was approximately $300 \mathrm{ml}$; no blood transfusions were needed. The postoperative outcome was remarkably uneventful, and the patient was discharged in the postoperative day 11 .

\section{DISCUSSION}

Cholangiocarcinoma has an incidence of about 3\% among gastrointestinal cancers (3). ICC is a distinct type of cholangiocarcinoma, along with the perihilar (cholangiocarcinoma arising at the level of the main biliary confluence) and distal (cholangiocarcinoma arising from the distal part of the common bile duct) types (4). ICC is the second most common malignancy of the liver, representing about $10 \%$ of all cholangiocarcinomas. The incidence in the Western world is approximately 1-2/ 100,000 $(5,6)$. ICC arises from the peripheral bile ducts within the liver parenchyma, proximal to the secondary biliary ducts. The majority of ICCS are adenocarcinomas, moderately differentiated, as our case.

The main risk factors for ICC are primary sclerosing cholangitis and fibro-polycystic liver disease, and chronic intrahepatic hepatolithiasis (7). Chronic liver disease (cirrhosis or chronic hepatitis, regardless of etiology) and metabolic disease (elevated waist circumference/ central obesity, dyslipidemia, hypertension, impaired fasting glucose) are now recognized as risk factors, particularly for intrahepatic cholangiocarcinoma $(1,8,9)$. Genetic conditions, such as Lynch syndrome and biliary papillomatosis, are also related to cholangiocarcinoma. Exposure to toxic chemicals used in radiology (certain contrast agents), auto, rubber, chemical, and wood-finishing industries are also correlated to ICC $(10,11)$. Parasite liver infections, in particular Opisthorchis viverrini and Clonorchis sinensis, are also significant risk factors for ICC (East Asia) (12).

For the diagnosis of ICC, MRCP has a diagnostic accuracy of up to $93 \%$ and is recommended for visualization of the tumor extension in the biliary ducts and vascular structures $(13,14)$. Indeed, MRCP proved to be more accurate in our case, depicting the centrally located ICC that passed undetected at CT; however, nor CT neither MRCP could correctly diagnosed the biliary thrombus, which was depicted as a type IIIB Klatskin tumor. Clinical utility of PET for diagnosing ICC in the liver when $\mathrm{CT}$ or MRI has been performed is limited (13). Despite these imaging modalities, as many as a third of patients with resectable disease on imaging have occult metastatic or locally advanced disease during diagnostic laparoscopy (15).

ICC is an aggressive cancer, with poor prognosis despite treatment, in comparison to other primary hepatic neoplasms (16), with the probability of cure as low as $10 \%$ (17). Surgical treatment is the only potentially curative treatment in patients with ICC. Liver resection remains the only option for a curative intent, with a median survival of less than 3 years. Because of the large size, central location and/or intraductal and periductal tumor infiltration, major hepatectomies are often deployed (up to $75 \%$ of cases) (18), requiring a bile duct resection and reconstruction in up to $25 \%$ of cases. Indeed, our case was included in the latter subgroup.

ICC is usually treated by liver resection with negative resection margins (19). Unfortunately, the resectability rate is low, less than $60 \%$, similar to the perihilar type (20). Moreover, among patients that benefit from liver resection, RO resection margins are usually obtained in only $20-40 \%(16,21)$, and even lower if larger margin as 5 -mm is considered curative $(22,23)$.

The traditional criteria for resectability of cholangiocarcinoma include absence of distant lymph node or liver metastases, absence of invasion of the main portal vein or main hepatic artery (although some centers perform en-bloc resection with vascular reconstruction), absence of extrahepatic adjacent organ invasion and of disseminated disease $(24,25,26)$. Resectability rates for cholangiocarcinomas increased over time, mainly due to aggressive operative strategies and broadened criteria for resectability. This trend includes vascular reconstruction and/or $0-\mathrm{mm}$ resection margin. In selected patients, ICC becomes resectable disease after downstaging by chemotherapy, but the risk of tumor progression under chemotherapy in borderline resectable presentations must be taken into consideration.

Multidisciplinary approach of ICC is difficult due to significant differences in disease course both across and within tumor stages, so often the therapeutic strategy is customized for each patient (4).

The presence of bile duct tumor thrombus associated to ICC is a very rare finding, being more common in case of hepatocellular carcinoma (2-9\%) $(27,28)$. Indeed, we found only 7 such former case reports after reviewing the literature $(29,30,31)$. In the most recent one, the authors choose to perform thrombectomy in order to avoid surgical complications, even though they admitted that extrahepatic bile duct resection and reconstruction are recommended for ICC. Another fact related to the tumor thrombus that deserves a comment is the normal bilirubin levels (and 
no episodes of jaundice in medical history) despite the extension of the thrombus into the common bile duct inducing dilatation of the entire intrahepatic biliary tree. This can only be explained by the very slow tumor thrombus progression that allowed in the same time bile to pass through and / or around it; the slow progression of the tumor is supported by the presence of significant atrophy of the left hemiliver, especially of the left lateral section (figs. 1 and 4).

The tumor involvement of all hepatic veins at the level of the hepatocaval confluence was in fact the criteria based on which the tumor could be considered non resectable. However, the patient was referred to liver resection with potential vascular resection and reconstruction of the RHV. However, the absence of RHV tumor infiltration at intraoperative ultrasound allowed the preservation of the RHV and the accessory middle right vein by accepting $0-\mathrm{mm}$ resection margin at this level. In this way, the vein resection and reconstruction were avoided, thus significantly decreasing the intraoperative and postoperative morbidity risk.

As reported in the literature, morbidity rates after resection for ICC exceeds $20 \%$, while mortality rates vary from $1 \%$ to $6 \%(32,33)$. Intraoperative and postoperative strategies, such as low central venous pressure, restricted fluid resuscitation improved recovery and decreased the risk of complications (34). It is worth mentioning that our case had a regular postoperative course, despite the major liver and bile duct resection.

While hepatocellular carcinoma (HCC) is commonly treated with liver transplantation, ICC is still controversial as such indication (35), with suboptimal results reported for ICC and combined HCC and ICC (36) consisting in $10-18 \%$ of estimated overall survival at 5 years (37). However, small ICC $(<2 \mathrm{~cm})$ may provide optimal results after liver transplantation (38).

Neoadjuvant chemotherapy (CHT) addresses occult metastatic disease and may provide tumor downstage facilitating liver resection, providing similar overall survival after curative-intent liver resection despite the more advanced presentation $(39,40)$. In our case, the patient was not referred to neoadjuvant $\mathrm{CHT}$ for several reasons. First, the patient was referred for surgery for a type IIIB Klatskin tumor, the ICC being initially undetected (at CT) and was diagnosed later at MRCP. After MRCP, the diagnose was simultaneous ICC and Klatskin tumor, situation that was considered difficult to deploy preoperative $\mathrm{CHT}$ due to technically challenging biopsies of the centrally located ICC and the allegedly Klatskin tumor. Moreover, the scenario of a delayed $\mathrm{CHT}$ due to these challenging biopsies and the risk that would not respond to $\mathrm{CHT}$, thus becoming irreversibly unresectable, was considered very probable and therefore avoided.

Adjuvant $\mathrm{CHT}$ is aimed to decrease the risk of disease recurrence, with similar regimen as the preoperative CHT (41). Unfortunately, the efficacy of CHT in cholangiocarcinoma is limited to a small subgroup of patients.

Established prognostic factors for ICC include age, CEA, CA19-9, vascular invasion, presence of lymph node metastases, direct invasion, local metastases, number of tumors, and tumor diameter and cirrhosis. Risk factors for survival after liver resection are age, margin status, number of tumors, tumor diameter, cirrhosis, lymph node metastases, and micro- or macrovascular invasion $(42,43,44)$.

\section{CONCLUSION}

In conclusion, ICC is a complex disease, with a dismal prognosis, usually diagnosed with locally advanced disease. Surgery usually consists in major liver resections that may improve survival in combination with chemotherapy but involves a significant risk of major postoperative morbidity and mortality. Locally advanced ICC is a challenging presentation for both diagnosis and treatment, for which complex major liver resection is effective when performed in a high volume HPB center.

\section{Conflict of interest}

All author declare that they have no conflict of interest.

\section{REFERENCES}

1. Shaib YH, El-Serag HB, Nooka AK, Thomas M, Brown TD, Patt YZ, et al. Risk factors for intrahepatic and extrahepatic cholangiocarcinoma: a hospital-based case-control study. Am J Gastroenterol. 2007;102(5):1016-21.

2. West J, Wood H, Logan RF, Quinn M, Aithal GP. Trends in the incidence of primary liver and biliary tract cancers in England and Wales 1971-2001. Br J Cancer. 2006;94(11):1751-8.

3. Vauthey JN, Blumgart LH. Recent advances in the management of cholangiocarcinomas. Semin Liver Dis. 1994;14(2):109-14.

4. Popescu I (coord). Textbook of hepatobiliary and pancreatic surgery and liver transplantation. Editura Academiei Române, Bucharest, 2016, ISBN 978-973-27-2592-4.

5. Singal AK, Vauthey JN, Grady JJ, Stroehlein JR. Intra-hepatic cholangiocarcinoma - frequency and demographic patterns: thirtyyear data from the M.D. Anderson Cancer Center. J Cancer Res Clin Oncol. 2011;137(7):1071-8.

6. Shin HR, Oh JK, Masuyer E, Curado MP, Bouvard V, Fang Y, et al. Comparison of incidence of intrahepatic and extrahepatic cholangiocarcinoma - focus on East and South-Eastern Asian Pac J Cancer Prev. 2010;11(5):1159-66.

7. Kim YT, Byun JS, Kim J, Jang YH, Lee WJ, Ryu JK, et al. Factors 
predicting concurrent cholangiocarcinomas associated with hepatolithiasis. Hepatogastroenterology. 2003;50(49):8-12.

8. Welzel TM, Graubard BI, Zeuzem S, El-Serag HB, Davila JA McGlynn KA. Metabolic syndrome increases the risk of primary liver cancer in the United States: a study in the SEER-Medicare database. Hepatology. 2011;54(2):463-71.

9. Zhou YM, Yin ZF, Yang JM, Li B, Shao WY, Xu F, et al. Risk factors for intrahepatic cholangiocarcinoma: a case-control study in China. World J Gastroenterol. 2008;14(4):632-5.

10. Sahani D, Prasad SR, Tannabe KK, Hahn PF, Mueller PR, Saini S. Thorotrast-induced cholangiocarcinoma: case report. Abdom Imaging. 2003;28(1):72-4.

11. Welzel TM, Graubard BI, El-Serag HB, Shaib YH, Hsing AW, Davila $\mathrm{JA}$, et al. Risk factors for intrahepatic and extrahepatic cholangiocarcinoma in the United States: a population-based case-control study. Clin Gastroenterol Hepatol. 2007;5(10):1221-8.

12. Anderson CD, Pinson CW, Berlin J, Chari RS. Diagnosis and treatment of cholangiocarcinoma. Oncologist. 2004;9(1):43-57.

13. Bridgewater J, Galle PR, Khan SA, Llovet JM, Park JW, Patel T, et al. Guidelines for the diagnosis and management of intrahepatic cholangiocarcinoma. J Hepatol. 2014;60(6):1268-89.

14. Suthar M, Purohit S, Bhargav V, Goyal P. Role of MRCP in differentiation of benign and malignant causes of biliary obstruction. $\mathrm{J}$ Clin Diagn Res. 2015:9(11):TC08-TC12.

15. Goere D, Wagholikar GD, Pessaux P, Carrère N, Sibert A, Vilgrain V, et al. Utility of staging laparoscopy in subsets of biliary cancers: laparoscopy is a powerful diagnostic tool in patients with intrahepatic and gallbladder carcinoma. Surg Endosc. $2006 ; 20(5): 721-5$

16. Razumilava N, Gores GJ. Cholangiocarcinoma. Lancet 2014 383:2168.

17. Spolverato G, Vitale A, Cucchetti A, Popescu I, Marques HP, Aldrighetti $\mathrm{L}$, et al. Can hepatic resection provide a long-term cure for patients with intrahepatic cholangiocarcinoma? Cancer. 2015; 121(22):3998-4006.

18. DeOliveira ML, Cunningham SC, Cameron JL, Kamangar F, Winter $\mathrm{JM}$, Lillemoe KD, et al. Cholangiocarcinoma: thirty-one-year experience with 564 patients at a single institution. Ann Surg. 2007; 245(5):755-62.

19. Dodson RM, Weiss MJ, Cosgrove D, Herman JM, Kamel I, Anders $\mathrm{R}$, et al. Intrahepatic cholangiocarcinoma: management options and emerging therapies. J Am Coll Surg. 2013;217(4):736-750.e4.

20. Nakeeb A, Pitt HA, Sohn TA, et al. Cholangiocarcinoma. A spectrum of intrahepatic, perihilar, and distal tumors. Ann Surg. 1996; 224(4):463-73; discussion 473-5

21. Spolverato G, Kim Y, Alexandrescu S, Popescu I, Marques HP, Aldrighetti $\mathrm{L}$, et al. Is Hepatic Resection for Large or Multifocal Intrahepatic Cholangiocarcinoma Justified? Results from a MultiInstitutional Collaboration. Ann Surg Oncol. 2015;22(7):2218-25.

22. Burke EC, Jarnagin WR, Hochwald SN, Pisters PW, Fong Y, Blumgart LH. Hilar Cholangiocarcinoma: patterns of spread, the importance of hepatic resection for curative operation, and a presurgical clinical staging system. Ann Surg. 1998;228(3):385-94.

23. Sakamoto E, Nimura Y, Hayakawa N, Kamiya J, Kondo S, Nagino M, et al. The pattern of infiltration at the proximal border of hilar bile duct carcinoma: a histologic analysis of 62 resected cases. Ann Surg. 1998;227(3):405-11.

24. Rajagopalan V, Daines WP, Grossbard ML, Kozuch P. Gallbladder and biliary tract carcinoma: A comprehensive update, Part 1. Oncology. Williston Park: 2004; 18:889.

25. Ebata T, Nagino M, Kamiya J, Uesaka K, Nagasaka T, Nimura Y. Hepatectomy with portal vein resection for hilar cholangiocarcinoma: audit of 52 consecutive cases. Ann Surg. 2003; 238(5):720-7.

26. Hemming AW, Reed Al, Fujita S, Foley DP, Howard RJ, et al. Surgical management of hilar cholangiocarcinoma. Ann Surg. 2005;
241(5):693-9; discussion 699-702.

27. Hu XG, Mao W, Hong SY, Kim BW, Xu WG, Wang HJ. Surgical treatment for hepatocellular carcinoma with bile duct invasion. Ann Surg Treat Res. 2016; 90(3):139-46.

28. Wang DD1, Wu LQ, Wang ZS. Prognosis of hepatocellular carcinoma with bile duct tumor thrombus after R0 resection: a matched study. Hepatobiliary Pancreat Dis Int. 2016;15(6):626-632.

29. I waki K, Kaido T, Yamamoto G, Kamo N, Yagi S, Taura K, et al. Massforming intrahepatic cholangiocarcinoma with portal vein tumor thrombus and bile duct tumor thrombus: A case report. Int J Surg Case Rep. 2017:40:13-16.

30. Lucidi V, Van Laethem JL, Sersté T, Gelin M, Donckier V. Peripheral cholangiocarcinoma with endoluminal tumor thrombus in main bile duct mimicking a Klatskin tumor. J Gastrointest Surg. 2008; 12(6):1149-50.

31. Yamamoto J, Kosuge T, Takayama T, Shimada K, Yamasaki S, Sakamoto M, et al. Intrahepatic cholangiocarcinoma presenting intrabile duct extension: clinicopathologic study of five resected cases. Jpn J Clin Oncol. 1997;27(1):18-21.

32. Endo I, Gonen M, Yopp AC, Dalal KM, Zhou Q, Klimstra D, et al. Intrahepatic cholangiocarcinoma: rising frequency, improved survival, and determinants of outcome after resection. Ann Surg. 2008;248(1):84-96.

33. de Jong MC, Nathan H, Sotiropoulos GC, Paul A, Alexandrescu S, Marques $\mathrm{H}$, et al. Intrahepatic cholangiocarcinoma: an international multi-institutional analysis of prognostic factors and lymph node assessment. J Clin Oncol. 2011;29(23):3140-5.

34. Kim Y, Ejaz A, Gani F, Wasey JO, Xu L, Frank SM, et al. Crystalloid administration among patients undergoing liver surgery: defining patient- and provider-level variation. Surgery. 2016:159(2):389-98.

35. Sapisochin G. Fernández de Sevilla E, Echeverri J, Charco R. Liver transplantation for cholangiocarcinoma: current status and new insights. World J Hepatol. 2015;7(22):2396-403.

36. DeOliveira ML. Liver transplantation for cholangiocarcinoma: current best practice. Curr Opin Organ Transplant. 2014;19(3): $245-252$.

37. Zhang XF, Bagante F, Chen Q, Beal EW, Lv Y, Weiss M, Popescu I, et al. Perioperative and long-term outcome of intrahepatic cholangiocarcinoma involving the hepatic hilus after curative-intent resection: comparison with peripheral intrahepatic cholangiocarcinoma and hilar cholangiocarcinoma. Surgery. 2018;163(5):1114-1120.

38. Rizvi S, Gores GJ. Pathogenesis, diagnosis, and management of cholangiocarcinoma. Gastroenterology. 2013;145(6):1215-1229.

39. Buettner S, Koerkamp BG, Ejaz A, Buisman FE, Kim Y, Margonis GA, et al. The effect of preoperative chemotherapy treatment in surgically treated intrahepatic cholangiocarcinoma patients - a multi-institutional analysis. J Surg Oncol. 2017;115(3):312-318.

40. Valle $\mathrm{J}$, Wasan $\mathrm{H}$, Palmer $\mathrm{DH}$, Cunningham $\mathrm{D}$, Anthoney $\mathrm{A}$, Maraveyas A, et al. ABC-02 Trial Investigators. Cisplatin plus gemcitabine versus gemcitabine for biliary tract cancer. N Engl J Med. 2010;362(14):1273-81.

41. Horgan AM, Amir E, Walter T, Knox JJ. Adjuvant therapy in the treatment of biliary tract cancer: a systematic review and meta-analysis. J Clin Oncol. 2012;30(16):1934-40.

42. Hyder O, Marques H, Pulitano C, Marsh JW, Alexandrescu S, Bauer TW, et al. A nomogram to predict long-term survival after resection for intrahepatic cholangiocarcinoma: an Eastern and Western experience. JAMA Surg. 2014;149(5):432-8.

43. Wang Y, Li J, Xia Y, Gong R, Wang K, Yan Z, et al. Prognostic nomogram for intrahepatic cholangiocarcinoma after partial hepatectomy. J Clin Oncol. 2013: 31(9):1188-95.

44. Hu LS, Weiss M, Popescu I, Marques HP, Aldrighetti L, Maithel SK, et al. Impact of microvascular invasion on clinical outcomes after curative-intent resection for intrahepatic cholangiocarcinoma. J Surg Oncol. 2019;119(1):21-29. 